\title{
A score of non-contrast transthoracic echocardiography to screen patent foramen ovale in patients with embolic stroke of undetermined source
}

Hui Zhang ${ }^{1 \dagger}$, Haiyan Tang ${ }^{2 \dagger}$, Fei Wu ${ }^{1}$, Chun Yu ${ }^{3}$, Qiang Dong ${ }^{1,4,5}$ and Wenjie Cao ${ }^{1, *^{*}}$

\begin{abstract}
Background: The aim of this study was to develop a screening score system of non-contrast transthoracic echocardiography (TTE) for patent foramen ovale (PFO) in patients with embolic stroke of undetermined source (ESUS).

Methods: We performed a retrospective analysis of 218 consecutive patients with a recent ESUS from 2015 to 2018, who received TTE and transcranial Doppler (TCD) as routine examinations. PFO was diagnosed by the bubble test of TCD. Significant differences of the non-contrast TTE findings and patient characteristics between PFO group and nonPFO group were selected into a score.

Results: PFO was diagnosed in 35.8\% (78/218) of the patients. Compared with non-PFO group, a larger median aortic root diameter (ARd) (34 mm vs. $32 \mathrm{~mm}, p=0.005)$, a lower median peak E wave velocity (Em) $(61.5 \mathrm{~cm} / \mathrm{s} \mathrm{vs.} 68 \mathrm{~cm} / \mathrm{s}$, $p=0.005)$ and a lower incidence rate of mitral regurgitation (34.6\% vs. 50.7\%, $p=0.022$ ) were seen in PFO group. $A R d>33 \mathrm{~mm}$ and $E \mathrm{~m}<72 \mathrm{~cm} / \mathrm{s}$ were the best thresholds to predict PFO in ROC analysis. A four-point score system (MEAD) including TTE criteria (including ARd $>33 \mathrm{~mm}, E \mathrm{~m}<72 \mathrm{~cm} / \mathrm{s}$ and without mitral regurgitation) and no history of diabetes predicted PFO with an area under curve of $0.67(95 \% \mathrm{Cl} 0.57-0.72, p<0.001)$. MEAD score $\geq 3$ was the best threshold to predict PFO with an accuracy of 0.64 (95\% Cl 0.57-0.7), a sensitivity of $0.65(95 \% \mathrm{Cl} 0.53-0.75)$ and a specificity of $0.63(95 \% \mathrm{Cl} 0.55-0.71)$.
\end{abstract}

Conclusion: The MEAD score measured with non-contrast TTE can be used to select patients for bubble test of TCD to increase the diagnostic yield of PFO after ESUS.

Keywords: Transthoracic echocardiography, Patent foramen ovale, Embolic stroke of undetermined source, Bubble test, Transcranial Doppler

\section{Background}

Embolic stroke of undetermined source (ESUS), which accounts for approximately $25 \%$ of cases and patent foramen ovale (PFO) is considered to be one of the major

\footnotetext{
*Correspondence: wenjiecao@fudan.edu.cn

${ }^{\dagger}$ Hui Zhang and Haiyan Tang are co-first authors.

${ }^{4}$ State Key Laboratory of Medical Neurobiology, Fudan University,

Shanghai, China

Full list of author information is available at the end of the article
}

causes of ESUS [1]. Ultrasonographic assessment of PFO, using bubble test with transthoracic echocardiograph (TTE), transesophageal echocardiography (TEE) and transcranial Doppler (TCD) remains the diagnostic approach of choice [2]. Although the prevalence of cerebrovascular complications after bubble test were reported very low, stroke risk from paradoxical microbubble embolization can be clinically significant and cannot be guaranteed [3]. Additionally, TTE and bubble test 
with TCD or TTE are still not routine examinations in most of the stroke centers. Thus, a safe and useful PFOscreening tool before contrast ultrasonographic assessment is important for clinical practice. In this study, we aimed to develop an easily measured screening tool by using non-contrast TTE for PFO in patients with ESUS.

\section{Methods \\ Patients}

We retrospectively reviewed patients diagnosed with acute ESUS from Jan 2015 to Jun 2018. ESUS was defined according to TOAST (Trial of Org 10,172 in Acute Stroke Treatment) classification. Those with data of both TTE and bubble test on TCD were included. Demographic characteristics, vascular risk factors (diabetes, hypertension, hyperlipidemia, smoking) and previous stroke history were documented. Ten-point Risk of Paradoxical Embolism (RoPE) score was calculated [4]. Brain magnetic resonance imaging or $\mathrm{CT}$ scan were reviewed to determine the locations of infarcts.

\section{Bubble test on TCD}

PFO was diagnosed by the bubble test on TCD [5]. TCD was done according to the Consensus Conference of Venice 15 by one of two experienced operators. A head frame was placed on the head to maintain the two $2 \mathrm{MHz}$ probes in place for monitoring bilateral middle cerebral artery (MCA) at a depth of $50-60 \mathrm{~mm} .9 \mathrm{~mL}$ isotonic saline solution, $1 \mathrm{~mL}$ of air, and 1 drop of the patient's blood were mixed through two $10 \mathrm{~mL}$ syringes connected by a three-way stopcock. The mixture was injected at least twice to assess the presence of microbubbles (MBs) in either MCA during the TCD monitoring. A first bolus was injected during normal respiration with the patient at rest. The procedure was repeated after a performance of strenuous Valsalva Maneuver by the patient. Right-toleft shunt (RLS) was quantified by counting the number of MBs within the first 3 cardiac cycles. More than $1 \mathrm{MBs}$ detected over a 25 -s post injection interval was considered to be positive contrast-enhanced TCD [6].

\section{TTE}

Parameters of TTE were obtained by retrospectively reviewing the reports. TTE were performed in the left lateral decubitus position using standard imaging planes according to the American Society of Echocardiography recommendations during hospitalization. The aorta root diameters (ARd) and left atria anteroposterior diameters (LAAPd), end-diastolic left ventricle diameters (EDLVd), end-systolic left ventricle diameters (ESLVd), left ventricle ejection fraction (LVEF), peak E-wave velocity (Em) and peak A-wave velocity (Am) were collected. Presences of mitral, tritral and aortic valve regurgitation were recorded.

\section{Statistical Analysis}

Statistical analyses were performed using SPSS, version 22 (SPSS Inc., Chicago, IL) and R software (https:// www.r-project.org). $P$ value less than 0.05 was considered to indicate statistical significance. Continuous variables were compared by Mann-Whitney U test and categorical variables were compared by Chi-square or Fisher's exact test between PFO and non-PFO groups. Multivariate regression including remarkable TTE findings and 10 individual items of RoPE score (including patient characteristics and age grades of per 10-year increase) was used to access the association of variables with PFO. Receiver operating characteristic (ROC) analysis was performed to determine the optimal threshold of remarkable continuous variables in predicting PFO. Base on the results, a 4-point score, including 4 independent criteria, was tested by ROC for predicting PFO. A 10-fold cross validation of the 4-score system was performed by using $R$ software.

\section{Results}

A total of 218 ESUS patients were finally included, (Fig. 1) median age was 53years (interquartile range [IQR], $39.75-60), 74.8 \%$ were male (163/218). PFO was detected in $35.8 \%(78 / 218)$ of the patients. No difference was found in age, sex, risk factors for stroke, infarct patten

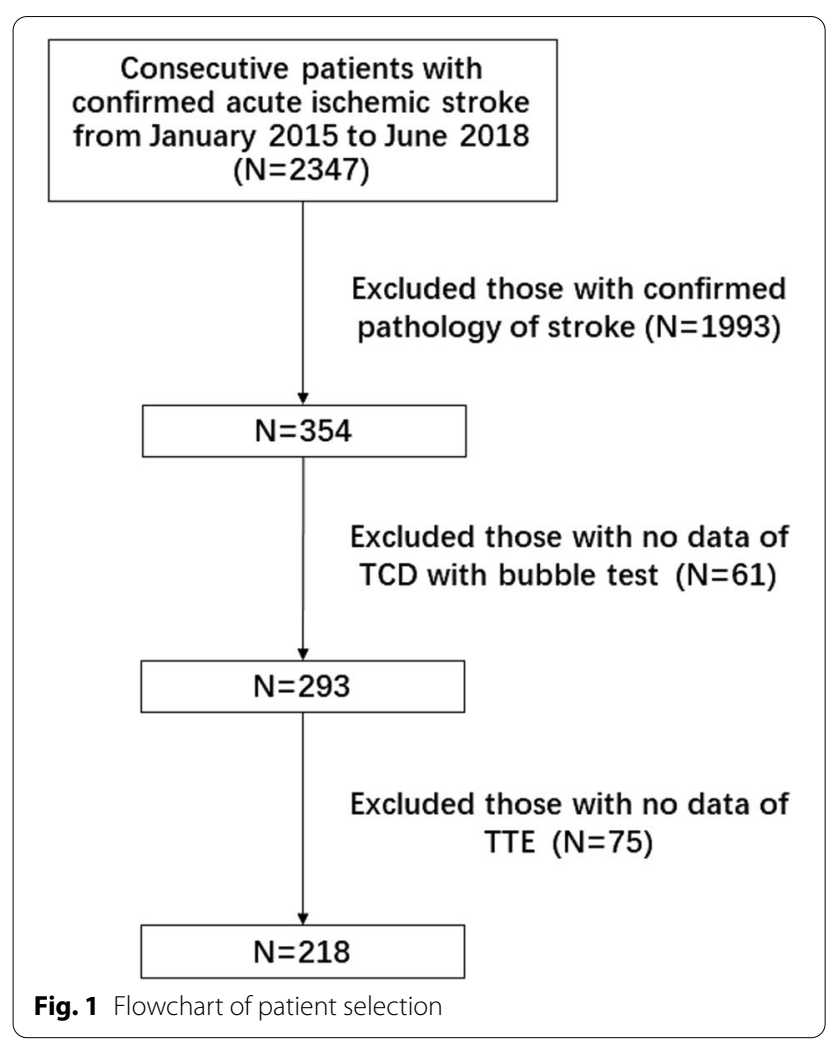


and RoPE score between PFO group and non-PFO group. In comparation of TTE parameters, a larger median ARd (measured at the level of the sinuses of Valsalva) $(34 \mathrm{~mm}$ vs. $32 \mathrm{~mm}, \mathrm{p}=0.005)$, lower median $\operatorname{Em}(61.5 \mathrm{~cm} / \mathrm{s}$ vs. $68 \mathrm{~cm} / \mathrm{s}, p=0.005)$ and lower incidence of mitral regurgitation ( $34.6 \%$ vs. $50.7 \%, p=0022)$ were found in the PFO group. (Table 1).

In ROC analysis, ARd predicted $\mathrm{PFO}$ with an area under curve (AUC) of 0.61 (95\%CI 0.53-0.69, $p=0.005$ ) (Fig. 1, in Supplement) and Em predicted PFO also with an AUC of 0.61 (95\%CI 0.53-0.69, $p=0.005$ ) (Fig. 2, in Supplement). Threshold of ARd and Em in predicting PFO was $33 \mathrm{~mm}$ and $72 \mathrm{~cm} / \mathrm{s}$ (analyzed with Youden index). In multivariate regression analysis, ARd $>33 \mathrm{~mm}$, $\mathrm{Em}<72 \mathrm{~cm} / \mathrm{s}$, without mitral regurgitation and no history of diabetes were significantly associated with PFO. (Table 2).

A 4-points score system (including without mitral regurgitation, $\mathrm{Em}<72 \mathrm{~cm} / \mathrm{s}$, ARd $>33 \mathrm{~mm}$ and no history of diabetes, MEAD) was derived to predict PFO. (Table 3) Based on the similarity of the odds ratios (OR), we assigned a single point for each of the 4 predictors in
MEAD score. (Table 2) MEAD score predicted PFO with an AUC of 0.67 (95\%CI 0.6-0.75, $p<0.001)$. (Fig. 3, in Supplement) The predictive values of each significance and score were list in Table 4. MEAD score $\geq 3$ was the best threshold to predict PFO with an accuracy of 0.64 (95\% CI 0.57-0.7), a sensitivity of 0.65 (95\% CI 0.53-0.75) and a specificity of 0.63 (95\% CI $0.55-0.71$ ). MEADs $\geq 2$ predicted PFO with the highest sensitivity of 0.91 (95\%CI $0.82-0.96$ ), while MEADs $=4$ predicted PFO with the highest specificity of 0.92 (95\%CI $0.87-0.96)$.

In 10-fold cross validation analysis, the mean AUC value of MEAD score was 0.67 in the prediction of PFO. (Table 1, in Supplement).

\section{Discussion}

We were able to develop a simple score to predict the detection of PFO in ESUS patients. The MEAD score measured with non-contrast TTE could be used to identify high-risk patients for PFO and reduce the use of bubble test. This allows the clinician to easily screen patients at highest risk of PFO for further confirming exams. Moreover, our data addressed the echocardiographic

Table 1 Univariate analysis between PFO group with non-PFO group

\begin{tabular}{|c|c|c|c|c|}
\hline Variable & $\begin{array}{l}\text { Total } \\
n=218\end{array}$ & $\begin{array}{l}\text { PFO } \\
n=78\end{array}$ & $\begin{array}{l}\text { Non-PFO } \\
n=140\end{array}$ & $P$ value \\
\hline Age, yr, median (IQR) & $53(39.75,60)$ & $52.5(43,59.25)$ & $53(38.25,61)$ & 0.846 \\
\hline Sex, male, \% (n) & $74.8(163)$ & $82.1(64)$ & $70.7(99)$ & 0.065 \\
\hline \multicolumn{5}{|l|}{ Clinical characteristics } \\
\hline Diabetes, \% (n) & $18.8(41)$ & $12.8(10)$ & $22.1(31)$ & 0.091 \\
\hline Hypertension, \% (n) & $40.4(88)$ & $42.3(33)$ & $39.3(55)$ & 0.663 \\
\hline Hyperlipidemia, \% (n) & $17.4(38)$ & $17.9(14)$ & $17.1(24)$ & 0.88 \\
\hline Smoking, \% (n) & $39.4(86)$ & $39.7(31)$ & $39.3(55)$ & 0.947 \\
\hline Previous TIA/stroke, \% (n) & $17.4(38)$ & $16.7(13)$ & $17.9(25)$ & 0.824 \\
\hline Cortical infarcts, \% (n) & $55.5(121)$ & $60.3(47)$ & $52.9(74)$ & 0.292 \\
\hline \multicolumn{5}{|l|}{ RoPE score } \\
\hline RoPE score, median (IQR) & $5(4,7.25)$ & $6(4,7)$ & $5(4,8)$ & 0.848 \\
\hline RoPE score >6, \% (n) & $36.2(79)$ & $30.8(24)$ & $39.3(55)$ & 0.21 \\
\hline \multicolumn{5}{|l|}{ TTE parameters } \\
\hline ARd, mm, median (IQR) & $33(31,36)$ & $34(31,36.25)$ & $32.5(30,35)$ & 0.005 \\
\hline LAAPd, mm, median (IQR) & $36(33,38)$ & $36(33,38)$ & $36(33,38)$ & 0.961 \\
\hline EDLVd, mm, median (IQR) & $49(46,52)$ & $48(45,53)$ & $49(46,52)$ & 0.759 \\
\hline ESLVd, mm, median (IQR) & $30(28,32)$ & $30(28,32)$ & $30(27,32)$ & 0.961 \\
\hline LEVF, \%, median (IQR) & $69(65,72)$ & $69(66,72)$ & $69(65.25,72)$ & 0.814 \\
\hline Em, cm/s, median (IQR) & $66(53,77.5)$ & $61.5(51,70.25)$ & $68(56,82)$ & 0.005 \\
\hline Am, cm/s, median (IQR) & $68(55,80)$ & $67.5(55.5,79)$ & $68.5(55,80)$ & 0.263 \\
\hline Mitral regurgitation, \% (n) & $45(98)$ & $34.6(27)$ & $50.7(71)$ & 0.022 \\
\hline Tritral regurgitation, \% (n) & $19.7(43)$ & $19.2(15)$ & $20(28)$ & 0.891 \\
\hline Aortic valve regurgitation, \% (n) & $20.6(45)$ & $23.1(18)$ & $19.3(27)$ & 0.507 \\
\hline
\end{tabular}

PFO patent foramen ovale, IQR interquartile range, $A R d$ aortic root diameter, RoPE Risk of Paradoxical Embolism, TTE transthoracic echocardiography, LAAPd left atria anteroposterior diameters, EDLVd end-diastolic left ventricle diameters, ESLVd end-systolic left ventricle diameters, $L V E F$ left ventricle ejection fraction, Em peak E wave velocity, $A m$ peak $\mathrm{A}$ wave velocity 
Table 2 Univariate and multivariate analysis of the predictors of PFO

\begin{tabular}{|c|c|c|c|c|}
\hline & \multicolumn{2}{|l|}{ Univariate } & \multicolumn{2}{|l|}{ Multivariate } \\
\hline & $\begin{array}{l}\text { OR } \\
(95 \% \mathrm{Cl})\end{array}$ & $P$ Value & $\begin{array}{l}\text { OR } \\
(95 \% \mathrm{Cl})\end{array}$ & $P$ Value \\
\hline \multicolumn{5}{|c|}{ Echocardiographic Characteristic } \\
\hline ARd $>33 \mathrm{~mm}$ & $1.99(1.13-3.5)$ & 0.016 & $2.28(1.18-4.39)$ & 0.013 \\
\hline $\mathrm{Em}<72 \mathrm{~cm} / \mathrm{s}$ & $2.26(1.22-4.18)$ & 0.009 & $2.23(1.08-4.59)$ & 0.029 \\
\hline Without mistral regurgitation & $1.94(1.09-3.44)$ & 0.023 & $2.11(1.14-3.93)$ & 0.017 \\
\hline \multicolumn{5}{|l|}{ Patient Characteristic } \\
\hline No history of hypertension & & & $0.88(0.45-1.71)$ & 0.71 \\
\hline No history of diabetes & & & $2.66(1.1-6.43)$ & 0.029 \\
\hline No history of stroke or TIA & & & $0.89(0.39-2.03)$ & 0.784 \\
\hline Nonsmoker & & & $1.43(0.73-2.79)$ & 0.291 \\
\hline Cortical infarct on imaging & & & $1.22(0.66-2.27)$ & 0.519 \\
\hline \multicolumn{5}{|l|}{ Age (y) } \\
\hline $18-29$ & & & $1.04(0.74-1.45)$ & 0.816 \\
\hline $30-39$ & & & $1.07(0.76-1.49)$ & 0.69 \\
\hline $40-49$ & & & $1.24(0.81-1.91)$ & 0.31 \\
\hline $50-59$ & & & $1.39(0.74-2.59)$ & 0.298 \\
\hline $69-69$ & & & $0.96(0.26-3.54)$ & 0.96 \\
\hline$* \geq 70$ & & & NA & NA \\
\hline
\end{tabular}

PFO patent foramen ovale, OR Odds ratio, ARd aortic root diameter, Em peak E wave velocity

* All the patients were younger than 70y in our study

Table 3 MEAD score

\begin{tabular}{lc}
\hline Characteristic & Points \\
\hline Without Mitral regurgitation & 1 \\
Em $<72 \mathrm{~cm} / \mathrm{s}$ & 1 \\
ARd $>33 \mathrm{~mm}$ & 1 \\
No history of Diabetes & 1 \\
Total score (sum of individual points) & 4
\end{tabular}

Em peak E wave velocity, ARd aortic root diameter changes of heart structures and functions in PFO patients, which could be useful to explore the potential mechanism of ESUS caused by PFO.

Dilatation of the aortic root may increase the risk of RLS by changing the angulation of the heart in such a way that flow streaming from the inferior vena cava into the right atrium is directed more towards the ostium secundum; thrombotic material is therefore more likely to cross into the systemic circulation, possibly causing

Table 4 AUC, sensitivity, specificity, PPV and NPV of each predictor and each threshold of MEAD score for the detection of PFO

\begin{tabular}{|c|c|c|c|c|c|}
\hline & $\begin{array}{l}\text { AUC } \\
(95 \% \mathrm{Cl})\end{array}$ & $\begin{array}{l}\text { Sensitivity } \\
(95 \% \mathrm{Cl})\end{array}$ & $\begin{array}{l}\text { Specificity } \\
(95 \% \mathrm{Cl})\end{array}$ & $\begin{array}{l}\text { PPV } \\
(95 \% \mathrm{Cl})\end{array}$ & $\begin{array}{l}\text { NPV } \\
(95 \% \mathrm{Cl})\end{array}$ \\
\hline \multicolumn{6}{|l|}{ Predictive power of each predictor } \\
\hline Without mitral regurgitation & $0.58(0.5-0.65)$ & $0.65(0.53-0.74)$ & $0.5(0.42-0.59)$ & $0.42(0.33-0.51)$ & $0.72(0.62-0.8)$ \\
\hline $\mathrm{Em}<72 \mathrm{~cm} / \mathrm{s}$ & $0.58(0.51-0.66)$ & $0.75(0.64-0.84)$ & $0.42(0.33-0.5)$ & $0.42(0.33-0.5)$ & $0.75(0.64-0.84)$ \\
\hline ARd $>33 \mathrm{~mm}$ & $0.58(0.5-0.66)$ & $0.52(0.4-0.63)$ & $0.64(0.55-0.72)$ & $0.45(0.34-0.55)$ & $0.7(0.62-0.78)$ \\
\hline No history of diabetes & $0.54(0.46-062)$ & $0.87(0.77-0.93)$ & $0.22(0.15-0.29)$ & $0.38(0.31-0.46)$ & $0.76(0.59-0.87)$ \\
\hline \multicolumn{6}{|c|}{ Predictive power of different MEADs thresholds } \\
\hline $\mathrm{MEADs} \geq 1^{*}$ & $0.5(0.42-0.58)$ & NA & NA & $0.35(0.29-0.42)$ & NA \\
\hline MEADs $\geq 2$ & $0.56(0.49-0.64)$ & $0.91(0.82-0.96)$ & $0.22(0.16-0.3)$ & $0.39(0.32-0.47)$ & $0.82(0.66-0.92)$ \\
\hline$M E A D s \geq 3$ & $0.64(0.56-0.72)$ & $0.65(0.53-0.75)$ & $0.63(0.55-0.71)$ & $0.5(0.39-0.6)$ & $0.76(0.67-0.84)$ \\
\hline MEADs $=4$ & $0.58(0.5-0.66)$ & $0.24(0.15-0.35)$ & $0.92(0.87-0.96)$ & $0.65(0.45-0.82)$ & $0.68(0.61-0.75)$ \\
\hline
\end{tabular}

AUC aera under curve, $P P V$ positive predictive value, NPV negative predictive value, $P F O$ patent foramen ovale, $A R d$ aortic root diameter, Em peak E wave velocity *All patients in our study were with MEADs $\geq 1$ 
a cryptogenic stroke. It has been reported that ARd, marked at the level of the sinuses of Valsalva $(34 \pm 4 \mathrm{vs}$ $31 \pm 3 \mathrm{~mm}, p<0.01$ ), is larger in PFO patients with cryptogenic stroke than in healthy people [7]. In this study, we compared the ARd in a ESUS patient cohort. All subjects had a homologous profile and our results consisted with previous study, demonstrated a larger median ARd of $34 \mathrm{~mm}$ in PFO patients than those without PFO.

E-wave velocity reflects the left atrial (LA)-left ventricle (LV) pressure gradient during early diastole and is affected by alterations in the rate of LV relaxation and LA. Elevated LA pressure is associated with the absence of RLS in AF stroke patients and may prevent opening of a PFO [8]. In our study, patients with PFO had lower Em suggest a decreased LA pressure, may associated with a RLS-related stroke. We explored that $\mathrm{Em}<72 \mathrm{~cm} / \mathrm{s}$ could be a mark in predicting PFO which has never been reported before.

Mitral regurgitation is the most common valvular heart disorder in high-income countries, and its prevalence increases with age [9]. In a large-scale cohort of UK adults with 10 years of follow-up, elevated blood pressure was continuously associated with an increased risk of mitral regurgitation [10]. Considering that the association between high blood pressure and mitral regurgitation is similar with that of arteriosclerosis stroke. ESUS patients with no mitral regurgitation may have fewer atherosclerotic risk factors, however, higher likelihood of PFO.

The RoPE score is a useful tool to assess the likelihood that the PFO is responsible for the event stroke. RoPE score was reported to identify PFO with an AUC of 0.68 [4]. However, RoPE score does not include echocardiographic features which could be more directly associated with PFO than other clinical features. In our cohort, there was no difference of RoPE score or proportion of RoPE $>6$ between PFO and non-PFO groups. In our study, the AUC of MEAD score in predicting PFO was 0.67, which is comparable to the RoPE score.

TEE and bubble test with TTE or TCD are current diagnostic approach of PFO, however, still not widely used in many hospitals due to their inconvenience. Noncontrast TTE is a convenient examination and routinely given to stroke patients for the assessment of cardiac function. The score system we derived based on noncontrast TTE findings can be easily used. MEADs $\geq 3$ predicted PFO with the highest accuracy of 0.64, MEADs $\geq 2$ predicted $\mathrm{PFO}$ with a high sensitivity of 0.91 , while MEADs $=4$ predicted PFO with a high specificity of 0.92 . By setting different thresholds, MEAD score can serve as a screening tool or a diagnostic tool, which may help doctors to screen patients for the next TEE or bubble test. It could be feasible to increase the positive rate of the following bubble test and reduce the overconsumption of clinical resources in clinical practice.

Several limitations of the present study need to be underlined. First, it was a single-center retrospective cohort study with a small sample size. Our score was built to estimate the probability of finding a PFO in ESUS patients, however, not to assess the likelihood that PFO is responsible for the stroke. Second, PFO was only diagnosed by the bubble test of TCD, not TEE. Although TEE was considered to be the standard technique for identifying a PFO, some patients were intolerant of this method. Finally, all patients in our study had a stroke. Thus, the differences between those with and without stroke in PFO were not examined. The score was not assessed to predict who will develop an ESUS, which needs to be addressed in further studies.

\section{Conclusions}

MEAD score is a risk score system based on non-contrast TTE parameters and patient characteristic that can easily be used to select patients for bubble test to increase the diagnostic yield of PFO after ESUS and might improve the secondary preventive strategy in order to prevent recurrent ischemic strokes.

\section{Abbreviations}

TTE: Transthoracic echocardiography; TEE: Transesophageal echocardiography; PFO: Patent foramen ovale; ESUS: Embolic stroke of undetermined source;

TCD: Transcranial Doppler; ARd: Aortic root diameter; Em: Peak E wave velocity; Am: Peak A wave velocity; RoPE: Risk of Paradoxical Embolism; MCA: Middle cerebral artery; MBs: Microbubbles; RLS: Right-to-left shunt; LAAPd: Left atria anteroposterior diameters; EDLVd: End-diastolic left ventricle diameters; ESLVd: End-systolic left ventricle diameters; LVEF: Left ventricle ejection fraction; ROC: Receiver operating characteristic; IQR: Interquartile range.

\section{Supplementary Information}

The online version contains supplementary material available at https://doi. org/10.1186/s12883-022-02565-w.

Additional file 1: Figure 1. ROC curve of aortic root diameter (ARd) for predicting PFO. Figure 2. ROC curve of peak E wave velocity (Em) for predicting PFO. Figure 3. ROC curve of MEAD score for predicting PFO. Table 1. 10-flod cross validation of MEAD score for predicting PFO.

\section{Acknowledgements \\ None.}

\section{Authors' contributions}

Conception and design: W.C. and Q.D. Analysis and interpretation of data: H.Z., H.T., F.W. and C.Y. Drafting the article: H.Z., H.T. and W.C. Critically revising the article: W.C. Reviewed submitted version of manuscript: all authors. Approval of the final version of the manuscript on behalf of all authors: W.C. Statistical analysis: W.C. and F.W. Study supervision: W.C. and Q.D.

\section{Funding}

This study was funded by National Natural Science Foundation of China (81901337) and National Natural Science Foundation of China (82071197). 


\section{Availability of data and materials}

The datasets generated and/or analysed during the current study are not publicly available due to limitations of ethical approval involving the patient data and anonymity but are available from the corresponding author on reasonable request.

\section{Declarations}

\section{Ethics approval and consent to participate}

The institutional ethics committee of the Huashan Hospital, Fudan University approved this study and waived informed consent based on the study design. We confirm that all methods were performed in accordance with the relevant guidelines and regulations.

\section{Consent for publication}

Not applicable.

\section{Competing interests}

We declare that we have no competing interests.

\section{Author details}

'Department of Neurology, Huashan Hospital, Fudan University, No.12 Wulumuqi Zhong Rd, Shanghai 200040, China. ${ }^{2}$ Department of Neurology and Institute of Neurology, Shuguang Hospital Affiliated to Shanghai University of Traditional Chinese Medicine, Shanghai, China. ${ }^{3}$ Department of Critical Care, West Campus of Huashan Hospital, Fudan University, Shanghai, China. ${ }^{4}$ State Key Laboratory of Medical Neurobiology, Fudan University, Shanghai, China. ${ }^{5}$ National Clinical Research Center for Aging and Medicine, Huashan Hospital, Fudan University, Shanghai, China.

Received: 22 September 2021 Accepted: 24 January 2022

Published online: 04 February 2022

\section{References}

1. Hart RG, Diener HC, Coutts SB, et al. Embolic strokes of undetermined source: the case for a new clinical construct. Lancet Neurol. 2014:13:429-38.

2. Pearson AC, Labovitz AJ, Tatineni S, Gomez CR. Superiority of transesophageal echocardiography in detecting cardiac source of embolism in patients with cerebral ischemia of uncertain etiology. J Am Coll Cardiol. 1991:17:66-72.

3. Romero JR, Frey JL, Schwamm LH, et al. Cerebral ischemic events associ ated with 'bubble study' for identification of right to left shunts. Stroke. 2009;40:2343-8.

4. Kent DM, Ruthazer R, Weimar C, et al. An index to identify stroke-related vs incidental patent foramen ovale in cryptogenic stroke. Neurology. 2013;81:619-25.

5. Komar M, Olszowska M, Przewlocki T, et al. Transcranial Doppler ultrasonography should it be the first choice for persistent foramen ovale screening? Cardiovasc Ultrasound. 2014;12:16.

6. Blersch WK, Draganski BM, Holmer SR, et al. Transcranial duplex sonography in the detection of patent foramen ovale. Radiology. 2002;225:693-9.

7. Keenan NG, Brochet E, Juliard JM, et al. Aortic root dilatation in young patients with cryptogenic stroke and patent foramen ovale. Arch Cardiovasc Dis. 2012;105:13-7.

8. Aoki J, Kimura K, Iguchi Y, et al. Higher LA pressure may prevent opening of patent foramen ovale in acute ischemic stroke patients with atria fibrillation. J Neurol Sci. 2011:304:111-6.

9. Andell P, Li X, Martinsson A, et al. Epidemiology of valvular heart disease in a Swedish nationwide hospital-based register study. Heart. 2017:103:1696-703

10. Rahimi K, Mohseni H, Otto CM, et al. Elevated blood pressure and risk of mitral regurgitation: A longitudinal cohort study of 5.5 million United Kingdom adults. PLoS Med. 2017;14:e1002404

\section{Publisher's Note}

Springer Nature remains neutral with regard to jurisdictional claims in published maps and institutional affiliations.

Ready to submit your research? Choose BMC and benefit from:

- fast, convenient online submission

- thorough peer review by experienced researchers in your field

- rapid publication on acceptance

- support for research data, including large and complex data types

- gold Open Access which fosters wider collaboration and increased citations

- maximum visibility for your research: over $100 \mathrm{M}$ website views per year

At BMC, research is always in progress.

Learn more biomedcentral.com/submissions 